Z Rheumatol 2020 $\cdot 79: 385-388$ https://doi.org/10.1007/s00393-020-00799-y Online publiziert: 27. April 2020 (c) Springer Medizin Verlag GmbH, ein Teil von Springer Nature 2020

Der gegenwärtige Ausbruch der severe acute respiratory syndrome coronavirus 2 (SARS-CoV-2) Infektion stellt uns alle vor beispiellose Herausforderungen. In der Betreuung unserer Patienten sehen wir uns zunehmend mit Fragen und Sorgen konfrontiert, auf die es kaum Antworten gibt. Dies macht die Interaktion nicht einfach, führt zu einem Gefühl der Unsicherheit, aber nicht zur Beruhigung, und schürt tendenziell Angst,

Die Autoren K. Krüger und C. Iking-Konert haben zu gleichen Teilen zum Manuskript beigetragen.

H. Schulze-Koops ${ }^{1,2} \cdot$ J. Holle ${ }^{3} \cdot$ F. Moosig ${ }^{3} \cdot$ C. Specker ${ }^{1,4} \cdot$ P. Aries ${ }^{5} \cdot$ G. Burmester ${ }^{6} \cdot$

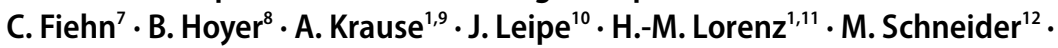
P. Sewerin ${ }^{12} \cdot$ A. Voormann ${ }^{1} \cdot$ U. Wager ${ }^{1,13} \cdot$ K. Krüger $^{14} \cdot$ C. Iking-Konert ${ }^{15} \cdot$ im $^{2}$ Auftrag der Kommission Pharmakotherapie und des Vorstandes der Deutschen Gesellschaft für Rheumatologie

' Deutsche Gesellschaft für Rheumatologie e. V., Berlin, Deutschland; ${ }^{2}$ Sektion Rheumatologie und Klinische Immunologie, Medizinische Klinik IV, Ludwig-Maximilians-Universität München, München, Deutschland; ${ }^{3}$ Rheumazentrum Schleswig-Holstein Mitte, Neumünster, Deutschland; ${ }^{4}$ Klinik für Rheumatologie und Klinische Immunologie, Kliniken Essen-Mitte, Essen, Deutschland; ${ }^{5}$ Rheumatologie im Struenseehaus, Hamburg, Deutschland; ${ }^{6}$ Klinik für Rheumatologie und Klinische Immunologie, Charité - Universitäts Medizin Berlin, Freie Universität und Humboldt Universität Berlin, Berlin, Deutschland; ' Medical Center Baden-Baden und ViDia-Kliniken Karlsruhe, Praxis für Rheumatologie und Klinische Immunologie, Baden-Baden, Deutschland; ${ }^{8}$ Abteilung für Rheumatologie, 1. Medizinische Klinik, Universitätskrankenhaus Schleswig-Holstein Campus Kiel, Kiel, Deutschland; ${ }^{9}$ Abteilung für Rheumatologie, Osteologie und Klinische Immunologie, Immanuel Krankenhaus Berlin, Berlin, Deutschland; ${ }^{10}$ Abteilung für Rheumatologie, Medizinische Klinik V, Universitätskrankenhaus Mannheim, Mannheim, Deutschland; "Abteilung für Rheumatologie, Medizinische Klinik V, Universitätsklinikum Heidelberg, Heidelberg, Deutschland; ${ }^{12}$ Poliklinik, Funktionsbereich und Hiller Forschungszentrum für Rheumatologie, Universitätsklinikum Düsseldorf, Heinrich-Heine-Universität Düsseldorf, Düsseldorf, Deutschland; ${ }^{13}$ Abteilung für Rheumatologie, Klinik und Poliklinik für Endokrinologie,

Nephrologie, Rheumatologie, Universitätsklinikum Leipzig, Leipzig, Deutschland; ${ }^{14}$ Rheumatologisches Praxiszentrum, Kommission Pharmakotherapie der DGRh e. V., München, Deutschland; ${ }^{15}$ III. Medizinische Klinik und Poliklinik Sektion Rheumatologie, Universitätsklinikum Hamburg-Eppendorf, Hamburg, Deutschland

\title{
Aktuelle
}

\section{Handlungsempfehlungen der Deutschen Gesellschaft für Rheumatologie für die Betreuung von Patienten mit rheumatischen Erkrankungen während der SARS-CoV-2/Covid 19-Pandemie}

sowohl bei Patienten als auch bei Ärzten. Wir alle wünschen uns, dass diese Unsicherheit, die wir sonst im medizinischen Alltag so selten wahrnehmen, bald ein Ende findet. Die Deutsche Gesellschaft für Rheumatologie e.V. (DGRh) hat ihre Kommission Pharmakotherapie beauftragt, in einem fortlaufenden Prozess aktuelle Empfehlungen für die Versorgung rheumatologischer Patienten zu erarbeiten, um Handlungshilfen für den Umgang mit der Therapie von Patienten mit entzündlich-rheumatischen Erkrankungen/systemischen Autoimmun- erkrankungen während der aktuellen Bedrohung durch das SARS-CoV-2-Virus bereit zu stellen.

Bisher gibt es für Handlungsempfehlungen bei der Betreuung und Behandlung von Patienten mit rheumatischen Erkrankungen in Verbindung mit der SARS-CoV-2/Covid-19-Pandemie keine evidenzbasierten Daten. Dies betrifft insbesondere die speziellen Auswirkungen der Infektion auf Rheumakranke sowie den Einfluss der immunsuppressiven oder immunmodulierenden Antirheumatika auf die Infektion. Die nachfolgen- 
Tab. 1 Wichtige grundsätzliche Risiko-

faktoren für eine Infektion bei Patienten mit rheumatischen Erkrankungen

Höheres Lebensalter

Multimorbidität, insbesondere vorbestehende Lungenerkrankung, Diabetes mellitus

Anamnese früherer schwerer Infektionen (z. B. Sepsis)

Dauertherapie mit Glucocorticoiden, insbesondere ab $5 \mathrm{mg} / \mathrm{Tag}$ (Risiko nimmt mit der Dauerdosis zu)

Therapie mit DMARDs und anderen Immunsuppressiva (Ausnahmen: Hydroxychloroquin, Sulfasalazin)

Hohe Aktivität der rheumatischen Grunderkrankung

Aktuelle oder weniger als 8 Wochen zurückliegende Cyclophosphamid-Therapie

Erworbene und angeborene Immundefekte, insbesondere:

Immunglobulin-Mangel $<4 \mathrm{~g} / \mathrm{dl}$ lgG Lymphopenie unter 500/ul, CD4-Zellen unter 200/ul

den Empfehlungen fußen dementsprechend auf einem von der DGRh erstellten Expertenkonsensus. Er stützt sich auf Analogien zum Vorgehen bei anderen, länger bekannten Virusinfektionen, auf theoretischen Überlegungen und auf bisher bekannten Daten und Fakten zur SARS-CoV-2-Infektion. Es versteht sich, dass in jedem Einzelfall ein Abweichen von diesen Empfehlungen sinnvoll sein kann. Die DGRh wird ihre Empfehlungen ständig aktualisieren und diese Aktualisierungen auf ihrer Homepage (www.dgrh.de) veröffentlichen. Es wird dringend empfohlen, dass sich jeder Arzt auch über neue Entwicklungen während des Verlaufs der Infektion und über neue therapeutische Algorithmen, die sich aus den laufenden klinischen Versuchen bei der SARS-CoV-2/Covid-19Pandemie ergeben können, informieren sollte, da sich hieraus stets Änderungen dieser Konsensusempfehlungen ergeben können.

Folgende allgemeine Empfehlungen werden gegeben:

\section{Vermeidung von Infektionen}

- Es gelten die vom Robert-KochInstitut für die Allgemeinbevölkerung und für speziell gefährdete
Personen beschriebenen und täglich aktualisierten Maßnahmen.

- Patienten mit rheumatischen Er-

krankungen haben unter bestimmten

Bedingungen ein erhöhtes Infektionsrisiko (- Tab. 1). Ob dies auch für SARS-CoV-2-Infektionen gilt, ist nicht bekannt. Ob eine COVID19-Erkrankung bei Patienten mit einer entzündlich-rheumatischen Erkrankung schwerer verläuft als bei nicht rheumatisch erkrankten Personen ist ebenso wenig bekannt wie die Antwort auf die Frage, ob die medikamentöse Immunsuppression ein zusätzliches Risiko für einen schweren Verlauf darstellt. Patienten mit rheumatischen Erkrankungen sollten daher Empfehlungen zur Kontaktvermeidung konsequent befolgen. Hierzu gehört auch, mit dem Arbeitgeber zu besprechen, inwieweit eine Kontaktvermeidung am Arbeitsplatz umsetzbar ist. Patienten können Atteste ausgestellt werden, dass sie eine immunsuppressive/ immunmodulierende Therapie erhalten, mit dem sie sich an Betriebsärzte/ Amtsärzte/Arbeitgeber wenden können (s. Link auf der Seite www.dgrh. de).

- Kontakte zwischen SARS-CoV-2 Infizierten und Rheumatologen, bzw. zwischen Infizierten und rheumatologischen Versorgungseinrichtungen sollten vermieden werden, bis die Infektion abgeklungen ist (in der Regel $>14$ Tage nach Symptomende)

- Notwendige Kontrollen zur Therapieund Krankheitsüberwachung sollten zwar sichergestellt sein, im Einzelfall muss aber zwischen dem Risiko durch (Haus-) Arztbesuche und dem Risiko durch fehlende Kontrollen abgewogen werden. So können bei Patienten in stabiler Krankheitseinstellung und mit bereits länger laufender Therapie vorübergehend längere Kontrollintervalle zur Vermeidung von Kontakten erwogen werden.

Die Behandlung der SARS-CoV-2-Infektion selbst sollte durch den Hausarzt (milde Fälle), einen Infektiologen, einen Pneumologen oder ggf. einen Intensiv- mediziner (schwere Fälle) gesteuert werden, wobei der Rheumatologe beratend verfügbar sein sollte.

\section{Medikamentöse anti- rheumatische Therapie im Kontext der aktuellen COVID- 19-Pandemie}

Ein generelles Pausieren oder eine generelle Reduktion der Immunsuppression wird nicht empfohlen, da die Pandemie voraussichtlich länger andauern wird und immunsupprimierte Patienten im Falle einer Therapiereduktion oder eines Aussetzens der Immunsuppression einem erhöhten Risiko von Rezidiven ausgesetzt wären. Ein solches Rezidiv bzw. ein Schub der rheumatischen Grunderkrankung erhöht zum einen das Infektionsrisiko (siehe - Tab. 1). Zum anderen zieht diese Destabilisierung die Notwendigkeit nach sich, die immunsuppressive Therapie wieder (und möglicherweise über das ursprüngliche Maß hinaus) zu intensivieren.

Immunsuppressive Therapien zur Remissionsinduktion (z. B. bei Vaskulitiden u. a.) sollten nicht verzögert oder unterdosiert werden, wobei etablierte Therapieregime mit geringeren Glucocorticoid (GC)-Dosen bevorzugt werden sollten.

Hydroxychloroquin (HCQ) sollte nicht abgesetzt werden, da dies möglicherweise eher nützlich als schädlich bei einer COVID-19-Infektion sein könnte.

Folgende spezielle Empfehlungen werden gegeben:

\subsection{Patienten ohne Infektzeichen}

- Zum Remissionserhalt eingesetzte immunsuppressive und/oder DMARD-Therapien sollten nicht allein aus Furcht vor einer Infektion mit SARS-CoV-2 beendet oder dosisreduziert werden. Dosisreduktionen von GC können im Einzelfall bei anhaltend stabiler Einstellung aber erwogen werden.

- Die Dosierungen der Immunsuppressiva bzw. DMARDs sollten wie üblich sorgfältig überprüft und ggf. korrigiert werden. Dies betrifft auch die in den Fachinformationen emp- 
fohlene Dosisanpassungen, z. B. bei Leukopenie.

\subsection{Patienten mit SARS-CoV-2- Kontakt aber ohne Infektzeichen}

- Fortführen der Therapie wie unter Punkt 2.1 beschrieben.

\subsection{Patienten mit SARS-CoV-2- Kontakt und Symptomen eines Infektes}

- Es sollte ein Abstrich auf SARS-CoV2 erfolgen.

- Bei leichten Symptomen und ohne Fieber sollte keine Therapieänderung erfolgen.

- Bei deutlichen Infektzeichen und insbesondere Fieber $\left(>38^{\circ} \mathrm{C}\right)$ sollte die anti-rheumatische Medikation pausiert werden.

- Eine etwaige GC-Dauertherapie sollte in gleicher Dosis fortgesetzt werden.

\subsection{Patienten positiv auf SARS-CoV-}

\section{2 getestet aber ohne Infektzeichen}

- Ein Pausieren oder Herauszögern einer ts- oder bDMARD-Therapie für die Dauer der mittleren Inkubationszeit sollte erwogen werden. Da häufig nicht bekannt ist, wann eine Infektion erfolgt ist, sollte eine Pause für 5-6 Tage nach Abstrich erwogen werden.

- Eine etwaige GC-Dauertherapie sollte in gleicher Dosis fortgesetzt werden.

- csDMARDs sollten nicht abgesetzt werden.

\subsection{Patienten positiv auf SARS-} CoV-2 getestet und Symptomen eines Infektes

- Die antirheumatische Medikation sollte pausiert werden.

- Eine etwaige GC-Dauertherapie sollte in gleicher Dosis fortgesetzt werden.

\section{Supportive Maßnahmen (alle Patientengruppen)}

- Entsprechend den Empfehlungen der STIKO sollte der Impfstatus

Z Rheumatol 2020 · 79:385-388 https://doi.org/10.1007/s00393-020-00799-y

(c) Springer Medizin Verlag GmbH, ein Teil von Springer Nature 2020

H. Schulze-Koops - J. Holle · F. Moosig · C. Specker · P. Aries · G. Burmester · C. Fiehn · B. Hoyer A. Krause - J. Leipe · H.-M. Lorenz - M. Schneider · P. Sewerin · A. Voormann - U. Wager · K. Krüger · C. Iking-Konert · im Auftrag der Kommission Pharmakotherapie und des Vorstandes der Deutschen Gesellschaft für Rheumatologie

\section{Aktuelle Handlungsempfehlungen der Deutschen Gesellschaft für Rheumatologie für die Betreuung von Patienten mit rheumatischen Erkrankungen während der SARS-CoV-2/Covid 19- Pandemie}

\section{Zusammenfassung}

In der aktuellen SARS-CoV-2 Pandemie bestehen viele Fragen mit Blick auf die sichere Behandlung von Patienten mit entzündlichrheumatischen Erkrankungen. Auf viele dieser Fragen gibt es zur Zeit noch keine evidenzbasierte Antwort und das macht die Betreuung der Patienten nicht leicht. Die Deutsche Gesellschaft für Rheumatologie e. V. (DGRh) will mit diesen ersten Empfehlungen Hilfestellung für spezielle Belange in der Betreuung von Rheumapatienten angesichts der aktuellen Bedrohung durch SARS-CoV-
2 geben. Um den dynamischen weltweiten Erkenntnisgewinn für unsere Patienten zu nutzen, werden die Empfehlungen regelmäßig aktualisiert. Die aktualisierten Versionen der Empfehlungen werden auf der Homepage der DGRh hinterlegt.

Schlüsselwörter

SARS-CoV-2 · COVID-19 - Therapiemanagement · Empfehlungen · Entzündliche Erkrankungen

\section{Current guidance of the German Society of Rheumatology for the care of patients with rheumatic diseases during the SARS-CoV- 2/Covid 19 pandemic}

Abstract

In the current SARS-CoV-2 pandemic there are many questions regarding the safe treatment of patients with inflammatory rheumatic diseases. Many of these questions cannot yet be answered on an evidence-based basis and this does not make patient care easy. The German Society for Rheumatology (DGRh) hopes that these initial recommendations will provide support for specific issues in the care of patients with inflammatory rheumatic diseases in view of the current threat posed by SARS-CoV-2. In order to take advantage of the dynamic worldwide gain in knowledge for our patients, the recommendations will be updated regularly. The updated versions of the recommendations are deposited on the homepage of the DGRh.

Keywords

SARS-CoV-2 $\cdot$ COVID-19 - Therapy Management - Recommendations · Inflammatory diseases aktualisiert werden (Schwerpunkt: Pneumokokken, Influenza).

- Eine PjP-Prophylaxe sollte bei entsprechender Indikation (v. a. Cyclophosphamidtherapie, GC $>15 \mathrm{mg}$ Prednisolonäquivalent) durchgeführt werden.

Diese Empfehlungen wurden von der Kommission Pharmakotherapie und dem Vorstand der Deutschen Gesellschaft für Rheumatologie erstellt (Stand 14.04.2020)

\section{Korrespondenzadresse}

\section{Prof. Dr. med. H. Schulze-Koops}

Sektion Rheumatologie und Klinische Immunologie, Medizinische Klinik IV, LudwigMaximilians-Universität München Pettenkoferstraße 8a, München, Deutschland Hendrik.Schulze-Koops@med.unimuenchen.de

\section{Prof. Dr. med. K. Krüger}

Rheumatologisches Praxiszentrum, Kommission Pharmakotherapie der DGRh e. V. St. Bonifatius-Straße 5, 81541 München, Deutschland

Klaus.Krueger@med.uni-muenchen.de 


\section{Einhaltung ethischer Richtlinien}

Interessenkonflikt. H. Schulze-Koops, J. Holle,

F. Moosig, C. Specker, P. Aries, G. Burmester, C. Fiehn, B. Hoyer, A. Krause, J. Leipe, H.-M. Lorenz, M. Schneider, P. Sewerin, A. Voormann, U. Wager, K. Krüger und C. Iking-Konert geben an, dass kein Interessenkonflikt besteht.

Für diesen Beitrag wurden von den Autoren keine Studien an Menschen oder Tieren durchgeführt. Für die aufgeführten Studien gelten die jeweils dort angegebenen ethischen Richtlinien.

\section{e.Medpedia: Die neue Online-Enzyklopädie für Ärzte}

e.Medpedia ist die neue digitale Enzyklopädie für Ärzte und ermöglicht das schnelle Nachschlagen auf Basis medizinischer Standardwerke von Springer. Die über Peer-ReviewVerfahren begutachteten Einträge werden von über 2.800 erfahrenen klinischen Experten verfasst und fortlaufend aktualisiert.

- e.Medpedia enthält alle Inhalte von über 20 etablierten Referenzwerken von Springer

- Inklusive unzähliger Abbildungen, klinischer Bilder, Tabellen und Schemata sowie Videos

- Verfasst von über 2.800 renommierten Fachärzten, gesichert durch Peer Review-Verfahren

- Komfortable Suchfunktion mit schneller Erkennung der Suchwörter

- Über 7.000 Querverlinkungen zwischen den einzelnen Einträgen

- Die bestehenden Einträge werden fortlaufend aktualisiert

-Weitere Fachgebiete werden kontinuierlich erweitert

- Mobile Nutzung über Smartphones - online und offline mit der e.Medpedia App für iOS- und Android-Geräte

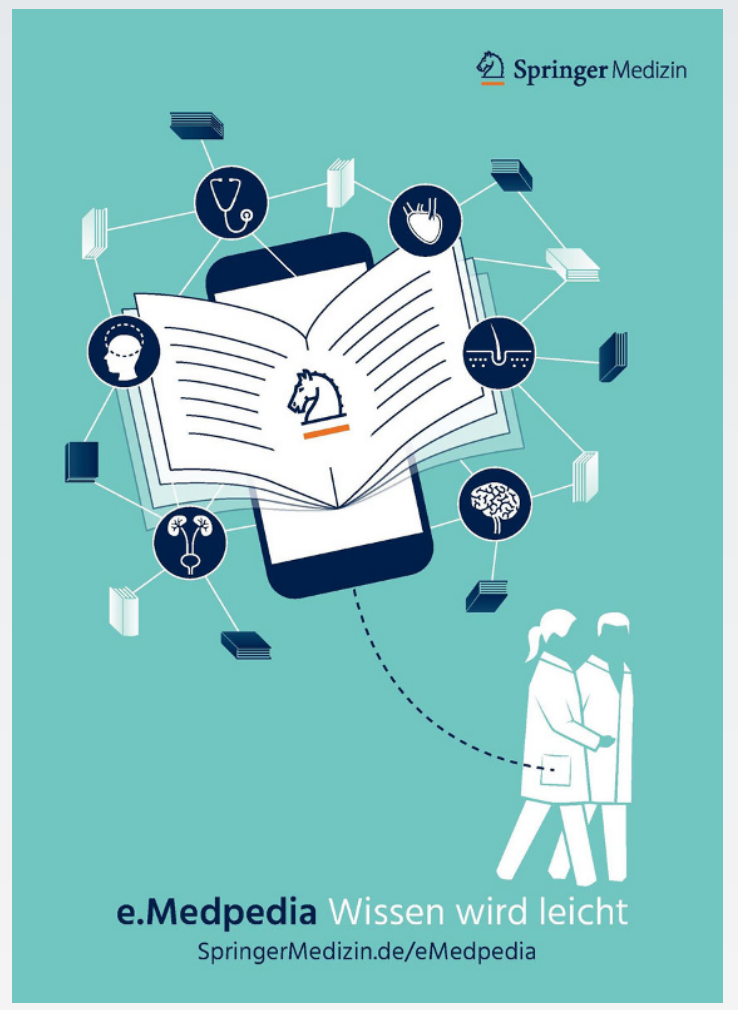

